\title{
The Prevalence of Bacterial Contamination in Transfusable Platelets in Lilongwe, Malawi
}

\author{
Grace Kusakala ${ }^{1}$, Pocha Saumel Kamdumula ${ }^{3}$ and Master Chisale ${ }^{1,2 *}$ \\ ${ }^{1}$ Biomedical Science Student, Mzuzu University, Mzuzu, Malawi \\ ${ }^{2}$ Laboratory Section, Mzuzu Central Hospital, Mzuzu, Malawi \\ ${ }^{3}$ URC Laboratory Project, Malawi
}

*Corresponding Author: Master Chisale, Biomedical Science Student, Mzuzu University and Laboratory Section, Mzuzu Central Hospital, Mzuzu, Malawi.

Received: August 12, 2019; Published: September 05, 2019

DOI: $10.31080 /$ ASMI.2019.02.0363

\begin{abstract}
Background: Throughout the world the prevalence of transfusion transmitted viral infections like HIV, hepatitis B and C have decreased, while that of transfusion transmitted infections due to bacterially contaminated blood products more especially platelets has increased. This burden has far much increased in the developing countries including Malawi. The prevalence of bacterial contamination in other countries like Zimbabwe and Ghana are $10.3 \%$ and $9 \%$ respectively, whereas in most of the developed countries it has been estimated to be $0.001 \%$ to $0.003 \%$.

This study aimed at establishing the prevalence of bacterial contamination in transfusable platelets at Malawi blood transfusion service (MBTS) in Lilongwe and identification of bacterial species that are most prevalent in the contamination of platelets pints.

Methods: Using a cross sectional study design, eighty three platelet units were randomly selected among those that were being stored at Malawi Blood Transfusion Service in Lilongwe. Within their storage period, these platelet pints were tested for bacterial contamination using blood and MacConkey agar at UNC laboratories in Lilongwe.

Results: Out of the total platelet units (83) that were tested for bacterial contamination, 3(3.61\%) were found positive, where $2(67.7 \%)$ units were tested positive on day five of its storage period and $1(33.3 \%)$ on day three of its storage period. The Staphylococcus aureus and Listeria monocytogenes were the bacteria isolated with $33.7 \%$ and $66.7 \%$ respectively.

Conclusion: The results of this study show that there is a considerable risk of transfusion transmitted bacterial infections in Malawi which needs to be urgently addressed. As such MBTS, all transfusion centers and laboratories in Malawi may need to adopt and implement measures which may check sterility as well as to reduce bacterial contamination of platelets units.
\end{abstract}

Keywords: Transfusion; Infections; Bacteria; Contamination; Platelets and Sepsis

\section{Introduction}

Bacterial contamination in platelet units is one of the most common long standing problem worldwide more especially in developing countries. This problem is a result of poor sterility mechanisms, lack of recognition and reporting of cases, failure to associate chills, rigors and fever which are signs and symptoms common in patients receiving platelet transfusion therapy with the possibility of transfusion of a bacterially contaminated platelet unit $[1,2]$. So far in Malawi there are very few or no reports from clinicians on transfusion associated reactions or sepsis which are as a result of the contamination of the transfusable platelets pints despite their demand. This has been hypothesized that bacterial contamination of donor platelets is significant but overlooked health hazard that may lead to hospital acquired infections in recipients.
Numerous studies have revealed that approximately 1 in 1000 platelet units may be contaminated with bacteria and that $10 \%$ to $40 \%$ of the recipients who receive bacterially contaminated platelet units are estimated to develop a life threatening transfusion transmitted sepsis [2,3]. Additionally, studIes in the United States,

\section{Methodology}

This was a quantitative cross sectional study [4] which was conducted in Lilongwe at MBTS due to the availability of the platelets in large quantities. The samples for this study were analyzed for bacterial contamination at University of North Carolina (UNC).

Sample size and sampling technique

The sample size for this study was 83 platelet pints, and it was derived according to the following formula: 
$\mathrm{n}=\mathrm{N} /\left[1+\mathrm{N}(\mathrm{e})^{2}\right]$

where $\mathrm{n}$ is the sample size $=83, \mathrm{~N}$ is the population size $=105$, and $\mathrm{e}$ is the level of precision $=0.05$.

The population size was driven by calculating an average from the total platelet pints that are issued out per month at MBTS in Lilongwe for an average period of four months; $2016 \mathrm{July}=91, \mathrm{Au}-$ gust $=103$, September $=136$, October $=87$ and November $=107$ platelet pints which was making an average of 105 .

This study used a non-probability random sampling technique [5]. All expired platelet units were excluded from this study.

Data collection tools, instrument and method

A checklist and a laboratory form was used, on which the data of each platelet units that was analyzed was entered together with laboratory results. A blood bank collection book which comprised of the platelet pack identities was also used.

Aliquots of sample for testing were taken from the platelet units using standard bacteriological safety and aseptic techniques. The platelet packs were thoroughly mixed. Some of the mixed plasma from the main bag was allowed to seep into the line of the pint where segments were made from each samples for testing were being taken from the platelet units. This was so as to maintain the closed system which is one of the international recommended aseptic techniques and is being practiced by the MBTS to prevent the blood products from contamination. After detaching each segment from the main bag, the segments were disinfected with $70 \%$ ethanol and with a sterile scissors the end of the tubes were cut off to draw out part of the sample for testing.

The testing for bacterial contamination was firstly done using gram stain, this was to determine the presence or to identify those platelet units that was heavily contaminated with bacteria. Aliquot was used for Gram stain and culture on agar media (blood and MacConkey agar plates) and incubated aerobically and anaerobically for $18-24$ hours at $37^{\circ} \mathrm{C}$ before reading of results. The culture was declared no growth after being incubated for at least 48 hours without any physical observable colony growth. The identities of bacteria growing on the culture plates were determined by colonial morphology, gram and spore stains; as well as standard biochemical tests $[6,7]$. All the laboratory work starting from gram stain, culturing and biochemical identification of bacterial species was done using the microbiology Standard Operating Procedure (SOP) used at UNC. Platelet units were tested for bacterial contamination on different days within their storage period.

\section{Ethical consideration}

The permission to conduct this study was sought from the Mzuzu University Ethical Committee through the department of Biomedical Sciences under the faculty of Health Sciences. Furthermore, permission to conduct this study was sought from the director of MBTS (Lilongwe). The data that was obtained from this study was treated with absolute confidentiality.

\section{Results and Findings}

A total of 83 platelet pints were tested for bacterial contamination and $3(3.61 \%)$ of the total proportion were tested positive. Two of the organisms that were isolated were gram positive and one gram negative.

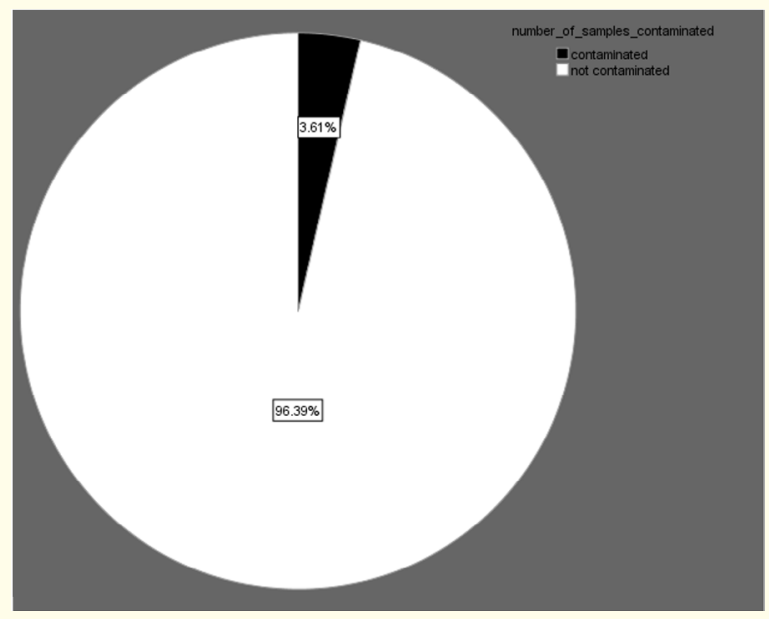

Figure 1: Bacterial contamination rate of platelet units.

A total of two gram positive bacterial species were isolated from the three platelet pints that were found to be contaminants. One platelet pint was contaminated by Staphylococcus aureus and two platelet pints were contaminated by Listeria monocytogenes.

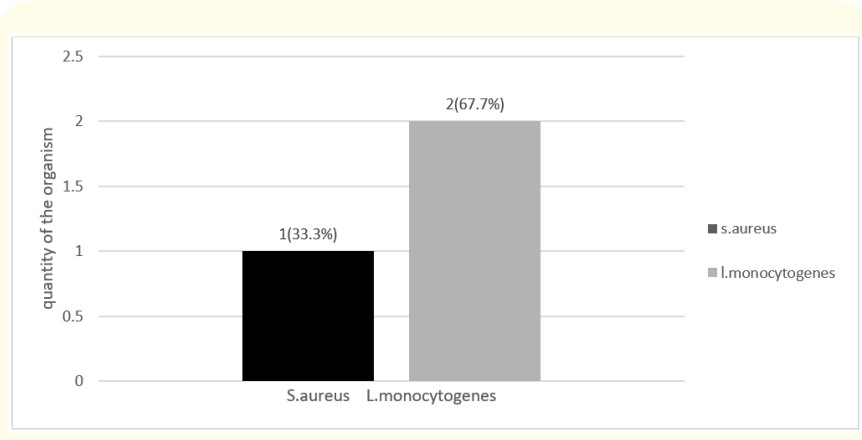

Figure 2: The prevalence of the isolated organisms. 
Day five seems to be the most prevalent day on which most platelet pints were contaminated, with the prevalence rate of $2(67.7 \%)$.

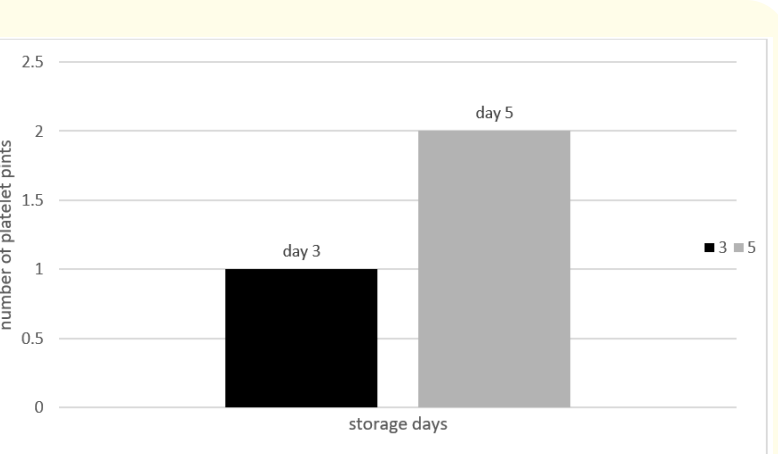

Figure 3: Storage days and the contamination rate.

\section{Discussion}

Bacterial contamination of blood products (platelets) has become one of the very critical but yet ignored problem in our society more especially in Africa particularly Malawi. Studies have shown that despite advances in strategies and efforts to address the risks associated with platelet transfusions and the harm it causes, the potential for bacterial contamination remains [8]. Women and children accounts for a large group of the recipients of these blood products as such they are at a greater risk of developing the transfusion transmitted infections [4]. Conducting studies pertaining to this topic in Africa particularly in Malawi might help to increase the knowledge on how to prevent and minimize the hazards or infections that arise due to the transfusion of bacterially contaminated products (platelets) through the delivery of safe blood to the recipients.

\section{Prevalence rate of bacterial contamination in platelet units}

This study aimed at determining the prevalence of bacterial contamination in transfusable platelets. Out of the 83 platelet units that were tested for bacterial contamination, the study managed to realize a prevalence of $3.61 \%$. Similar studies that were also conducted in some parts of Africa such as Zimbabwe and Ghana found a prevalence of $10.3 \%$ and $9 \%$ respectively $[4,9]$. It is possible that this study found the prevalence of bacterial contamination to be a bit lower than other studies conducted in other parts of Africa due to some different reasons. These reasons may include the difference in the techniques that were used in the isolation of the bacterial contaminants. This study used traditional culturing (blood and Mac Conkey agar) while other studies used advanced techniques like BacT/ALERT and Polymerase Chain Reaction (PCR) to detect bacterial contamination $[1,10]$.
In developed countries like United Kingdom, Japan and the United States of America, the prevalence seems to be much lower with the prevalence rate ranging from $0.001 \%$ to $0.003 \%$ [1]. This simply shows that the problem of bacterial contamination in platelet units is more severe in most African developing countries. This might be due to some factors which may include but not limited to that most African countries are not conducting rapid bacterial contamination detection tests prior to transfusion due to lack of knowledge on the prevalence of bacterial contamination of transfusable platelets in their countries. Another contributing factor might be due to the lack of resources as a result of financial crisis in most of the developing countries like Malawi and lastly in availability of sterility techniques in most developing countries.

The prevalence rate of bacterial contamination of platelet products in most of the developed countries seems to be low as compared to most of the developing countries due to a lot of measures. These measures may include that most of the developed countries have managed to come up and implement ways that inhibits and inactivates the growth of bacteria in platelet units [1]. These inhibitors are inserted in the platelet collection bag so that if by any means a bacteria enters the collection bag, it should be neutralized. Another contributing factor is that most of these countries have implemented the use of rapid bacterial contamination check prior to transfusion.

The prevalence rate in this study seems to be a bit lower than that of other studies that has been conducted in the surrounding parts of Africa. This might be due to the kind of laboratory method used, for example this study used media (blood and MacConkey agar) for culturing the bacterial contaminants in the platelet units. This method is considered to be less sensitive as compared to other methods $[1,10]$. This might be a limiting factor in such a way that this method could have missed some of the potential bacterial contaminants. Some studies conducted in other countries used advanced methods of detecting bacterial contaminants such as Polymerase Chain Reaction (PCR) or BacT/ALERT [1,10]. These methods are considered to be more sensitive in such a way that they are able to capture a variety of bacterial species.

There is a likelihood to have a high prevalence rate in Malawi if a different method were to be used. Knowing this, a similar study can also be conducted in Malawi using a different method such as BacT/ALERT or PCR just to bring to light if the prevalence might be higher than found using the method used in this study. 


\section{Bacterial isolates}

Over the years, different species of bacteria have been isolated out of the platelet products. This study managed to isolate two species of bacteria which were all gram positive. These bacteria were Staphylococcus aureus and Listeria monocytogenes. Some studies revealed that the gram positive organisms causes most of the septic reactions after transfusion of bacterially contaminated units [1].

Other studies have suggested that the isolation of Staphylococcus aureus is an indication that the platelet pint might have been contaminated by the skin flora during the collection of the product [1]. Listeria monocytogenes being one of the rare but widely distributed contaminant has also been one of the isolates in other similar studies $[3,7]$.

Listeria monocytogenes causes a serious infection called Listeriosis. This disease basically affects pregnant women, newborns, the elderly and those people who are immune compromised [11]. These groups of people being the most likely to be transfused with blood products like platelet are at a greater risk of developing this infection. One in five people with this infection die and when it affects pregnant mothers it leads to miscarriage, still birth, or the dearth of the newborn since it can pass through the placenta. The CDC further says that Listeriosis can lead to $20 \%$ of fetal loss during pregnancy and $3 \%$ death cases of the newborn [3].

This therefore shows that contamination of blood products (platelets) is a problem which need to be addressed to avoid and minimize the severity of the suggested risks of morbidity and mortality. As suggested by researchers, some of the preventative ways may include the implementation of rapid bacterial contamination check prior to transfusion. This study did not manage to as far as establishing the sources of the bacterial contamination which other studies suggested that it might be due donor bacteremia, to the inappropriate disinfection of the skin, skin core removal by the collection needle, contamination of the collection bags and poor storage condition $[4,7]$. This is why it is recommended that other studies should be conducted in Malawi so as to determine the sources of bacterial contamination.

\section{Storage days and platelet contamination}

Numerous studies have been conducted throughout the world with the aim of establishing the effect of storage duration in relation to the safety of platelet products. This study also aimed at discovering the most prevalent day where platelet products are most likely to be found contaminated. On the same note, this study found out that $2(67.7 \%)$ of the total contaminated platelet pints were contaminated on the fifth day of its storage period. The results of this study are also similar to other studies, who found out that $75 \%$ of the platelet products that were contaminated on the fifth day of their storage period [4].

Platelet storage at room temperature is said to be posing a greater risk as far as its safety is concerned [12]. Bacterial species are said to readily proliferate at room temperature, hence the longer the storage period the greater the risk of contamination [1214]. Room temperatures provides a conducive environment for the growth and multiplication of most bacterial species [12]. In trying to minimize this problem other countries have further reduced their platelets storage period from five days to three days [15].

This study encountered a lot of challenges but despite that there are some strong areas it managed to achieve and this include the fact that this study is the first of its kind in Malawi. The limiting factors includes the culturing method that this study used. These methods are considered to be less sensitive. Another limiting factor is that this study used a small sample size and therefore the results may not be generalized.

In conclusion, the results obtained from this study suggests that blood products (platelet) recipients in Malawi might be at a risk of developing transfusion transmitted bacterial infections. Other studies have suggested that the implementation of rapid bacterial contamination check and pathogen inactivation prior to transfusion reduces the risk of transfusion transmitted bacterial infections. It may also be helpful if there can be a critical linkage between the laboratory and clinical wards for rapid administration of plates since the quicker the administration the lower the risk of the contaminations. Adoption and implementation of these methods might be of great help to Malawi as far as this problem is concerned.

\section{Acknowledgement}

We are proudly thankful to Mzuzu University (Department of Biomedical Sciences), The Director of MBTS Lilongwe and staff and also lab manager and The Microbiology Department staff of UNC Lilongwe for their constructive criticism and support on this study.

Lastly, this study would not have been possible without the financial help from Hsin Lee under Luke International. 


\section{Bibliography}

1. Palavecino Elizabeth L., et al. Detecting Bacterial Contamination in Platelet Products (2014).

2. Advancing Transfusion and Cellular Therapy Worldwide Task Force. "Bacterial Contamination of Platelets: Summary for Clinicians on Potential Management Issues Related to Transfusion Recipients and Blood Donors". Advancing Transfusion and Cellular Therapy Worldwide (2005).

3. Burns Kathleen H and Jochewed B Werch. "Bacterial Contamination of Platelet Units". 128 (2004): 279-281.

4. Makuni Ngonidzashe., et al. "Brief Original Article Prevalence of Bacterial Contamination in Blood and Blood Products at the National Blood Service Zimbabwe". The Journal of Infection in Development Countries (2015): 2-5.

5. Girgis Samia A., et al. "Rapid Detection of Bacterial Contamination in Platelet Concentrates, by Polymerase Chain Reaction and DNA Sequencing in Comparison to Conventional Automated Culture". 3.4 (2014): 38-52.

6. Rood Ineke GH., et al. "Distribution, Origin and Contamination Risk of Coagulase-Negative Staphylococci from Platelet Concentrates". Journal of Medical Microbiology (2011): 592-999.

7. Bolarinwa Rahman A., et al. "Bacterial Contamination of Blood and Blood Components in a Tertiary Hospital Setting in Nigeria”. (2009): 1-6.

8. Levy Jerrold H., et al. "Bacterial Contamination of Platelets for Transfusion: Strategies for Prevention". Critical Care 22.1 (2018): 1-8.

9. Adjei Andrew Anthony., et al. "Bacterial Contamination of Blood and Blood Components in Three Major Blood Transfusion Centers, Accra, Ghana". 10 (2009): 265-269.

10. Mohammadi Tamimount., et al. "Detection of bacteria in platelet concentrates: comparison of broad-range real-time $16 \mathrm{~S}$ rDNA polymerase chain reaction and automated culturing". Automated Culturing 45 (2005): 731-736.

11. Control ITS. Listeria Monocytogenes (2001): 1-4.

12. Hillyer Christopher D., et al. "Bacterial Contamination of Blood Components: Risks, Strategies, and Regulation". (2003): 575589.
13. Centers America's Blood. "Bacterial contamination of blood components". Blood Bulletin 3.2 (2000).

14. Singhal Manmohan., et al. "Research Paper A Research Analysis on Blood Component Usage and Wastage in Blood Bank and Blood Component Center". Journal of Physiology and Pathophysiology 4 (2013): 23-28.

15. Schmidt M. Bacterial Contamination of Blood Products (2013): 177-180.

Volume 2 Issue 10 October 2019

(C) All rights are reserved by Master Chisale., et al. 